\title{
Exploring Constrained Type-2 Fuzzy Sets
}

\author{
Pasquale D'Alterio and Jonathan M. Garibaldi \\ School of Computer Science \\ University of Nottingham
}

\author{
Amir Pourabdollah \\ School of Science \& Technology \\ Nottingham Trent University
}

\begin{abstract}
Fuzzy logic has been widely used to model human reasoning thanks to its inherent capability of handling uncertainty. In particular, the introduction of Type-2 fuzzy sets added the possibility of expressing uncertainty even on the definition of the membership functions. Type- 2 sets, however, don't pose any restrictions on the continuity or convexity of their embedded sets while these properties may be desirable in certain contexts. To overcome this problem, Constrained Type-2 fuzzy sets have been proposed. In this paper, we focus on Interval Constrained Type-2 sets to see how their unique structure can be exploited to build a new inference process. This will set some ground work for future developments, such as the design of a new defuzzification process for Constrained Type-2 fuzzy systems.
\end{abstract}

\section{INTRODUCTION}

One of the main characteristics of fuzzy sets (FS), introduced in 1965 by Zadeh [1], was the possibility of using them to build systems that could be designed in a way that was very close to human reasoning. The introduction of the membership degrees as values in $[0,1]$, made the modelling of fuzzy systems (FSMs) easy even for non-computer scientists nor logicians thanks to the possibility of "computing with words" [2]. In fact, in many contexts, it is possible to model membership functions (MFs) on the basis of concepts expressible with words. A typical example is given by the height, from which we could extract MFs from human concepts such as low, medium and high.

One of the things that makes "computing with words" possible, is the fuzziness of the concepts that can be modelled through the MFs. However, the amount of uncertainty that can be handled by a Type-1 (T1) set, i.e. 'standard' fuzzy logic, is limited. Specifically, there is no uncertainty about the membership degrees at all, so in that sense, T1 fuzzy logic is very crisp. For this reason, Zadeh himself [3] introduced Type-2 (extendable to Type- $n$ ) fuzzy sets. They provide a higher degree of fuzziness by taking the uncertainty to the level of the definition of the MFs. In fact, in Type-2 FSMs, the membership degree is no longer a (crisp) value in $[0,1]$ but a T1 fuzzy set instead.

Unfortunately, the capability of handling more uncertainty comes at a cost: $\mathrm{T} 2$ sets require three dimensions to be represented, which makes them hard to draw, and all of the main operations on them have a higher computational cost compared to their T1 counterpart. Recently, a specific kind of T2 sets have been commonly used in real applications: interval T2 (IT2) [4] fuzzy sets. They pose some mathematical limitations on the third dimension of a T2 set and therefore identify a subset of all the possible general T2 sets. Specifically, the value of the third dimension is set to be one for all the points in the support set and zero for the others. By doing this, the third dimension can be dropped and the IT2 set is fully identified by its footprint of uncertainty (FOU), i.e. the area contained between the lower-bound and upper-bound MFs. (Fig.1).

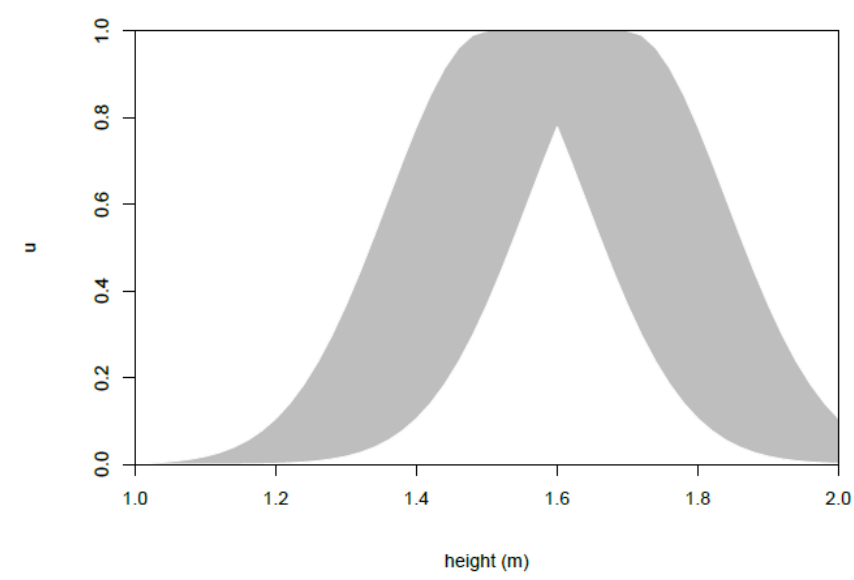

Fig. 1. Possible FOU for an IT2 Gaussian representing the concept of medium height (picture from [5])

Another key notion in fuzzy set theory is the concept of embedded set (ES). Intuitively, they represent (in the T2 case) a possible path along the FOU of the set they belong to. Even though they are widely used for many operations such as the centroid defuzzification [6], there isn't a clear relationship between the shape of the embedded set and the upper-bound or lower-bound functions.

In [5] the authors claim that the general definition of a T2 or IT2 FS, is too generic in certain contexts since there are no conditions on the MFs. As a consequence, the resulting FOUs and ESs that can be completely unrelated to the modelling of the concepts that they should represent. To overcome these limitations, they introduce a new kind of set, named Constrained Type-2 (CT2) fuzzy sets, by imposing the concepts of shape coherency and explicit variability on the FOU and the ES.

A similar concept was also introduced in [7]. In this paper the author defines a new kind of T2 FS in which only the nonconvex and normal embedded sets are considered acceptable. On the basis of that, a new constrained representation theorem and defuzzification process is derived.

The aim of this work is to explore this new kind of fuzzy sets, in order to establish some ground work for future 
developments. Being an introductory paper, we focused on the intuitive idea behind the generation, use and inference process of CT2 sets rather than the formalization of their properties and mathematical definitions, which will be carefully addressed in future works.

\section{PRELIMINARIES}

In this section we will provide some formal definition of fuzzy concepts that will be used throughout the paper.

Definition 1. A Tl FS is characterised by a Tl MF:

$$
\mu_{\widetilde{A}}: X \rightarrow \underset{U}{[0,1]}
$$

with $X$ being the primary domain and $U$ being the primary membership of $x$

Definition 2. A T2 FS is characterised by a T2 MF:

$$
\widetilde{\mu}_{\widetilde{A}}: X \times \underset{U}{[0,1]} \rightarrow \underset{V}{[0,1]}
$$

$X$ still represents the primary domain while $U$ is the secondary domain and $V$ the secondary membership of $x$.

Definition 3. An IT2 FS is characterised by an IT2 MF:

$$
\mu_{\widetilde{A}}^{I}: X \rightarrow \underset{U}{L([0,1])}
$$

In this case, $X$ still is the primary domain, $L([0,1])$ denotes the set of all the subset in the closed interval $[0,1]$ and $U$ denotes the primary membership of $x$.

Definition 4. A T2 ES is a path along the T2 set it belongs to. It contains only one primary degree $u_{x}$ for each $x$, with its associated secondary grade $v_{x}$ :

$$
\widetilde{\mu}_{\widetilde{A}}\left(x, u_{x}\right)=v_{x} \forall x \in X
$$

Definition 5. A T1 ES represents a projection of a T2 ES, i.e. its secondary degree has been dropped. Therefore it contains one primary degree $u_{x}$ for each $x$ :

$$
\mu_{\widetilde{A}}(x)=u_{x} ; \widetilde{\mu}_{\widetilde{A}}\left(x, u_{x}\right)=v_{x} \forall x \in X
$$

Definition 6. A similarity relation $\delta: X \times X \rightarrow[0,1]$ is a fuzzy relation [8] with the following properties:

Reflexive: $\delta(x, x)=1 ; \forall x \in X$

Symmetric: $\delta(x, y)=\delta(y, x) ; \forall x, y \in X$

T-Transitive: $T(\delta(x, y), \delta(y, z)) \leq \delta(x, z) ; \forall x, y, z \in X$ with $T$ being a T-norm

\section{CONSTRAINED TYPE-2}

The main idea behind the introduction of CT2 sets is that a general T2 FS has no constraints on the shape of its ES and its boundary functions. However, in some contexts, imposing some properties such as continuity or convexity is desirable. Because of the unconstrained definition of general T2 sets, as per definition 2, there is no relation between the ESs, FOU and the concept they should model in some contexts.

For example, if we imagine designing a Gaussian T1 MF to represent medium height, we might obtain something like the MF shown in Fig. 2.

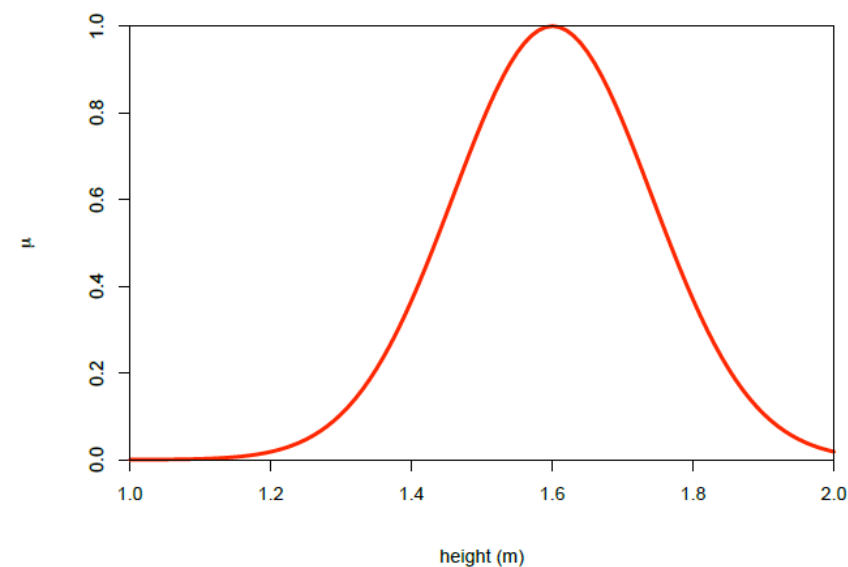

Fig. 2. Example of a T1 Gaussian modelling the concept of medium height (picture from [5])

This T1 set can then be used to design a T2 set. One strategy, would be to ask different people to take the T1 Gaussian and to place it accordingly to their idea of medium height. The result of this approach, would likely be something very similar to the set of T1 sets shown in Fig. 3.

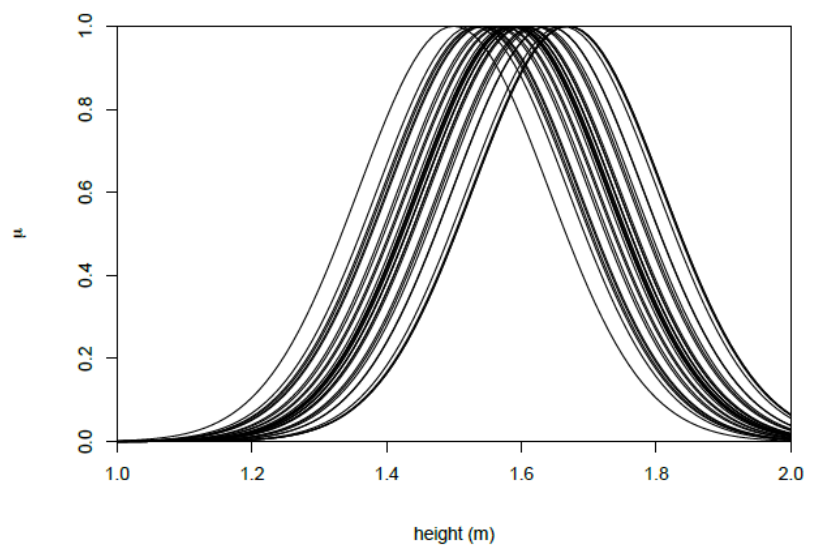

Fig. 3. Non-stationary Gaussian T1 set for the concept medium height (picture from [5])

Using the left-most and right-most T1 Gaussians to shape the FOU, however, would still generate a T2 set with no relation with the original Gaussian shape. Additionally, an ES such as the one shown in Fig. 4 would be acceptable in the standard $\mathrm{T} 2$ representation even though it has very little meaning for the concept of medium height that it is representing.

The sets shown in Fig. 3 can actually be seen as a nonstationary T1 set [9] (i.e. a set that is perturbed over time). The intuitive idea is to use the non-stationary representation to design a T2 set and by doing so, preserving the relation with the T1 MF of the concept we are modelling.

Informally, a CT2 set can be seen as the union of an infinite number of $\mathrm{T} 1$ sets obtained by shifting, in a finite interval, the original T1 set we used to model the concept. The latter set 


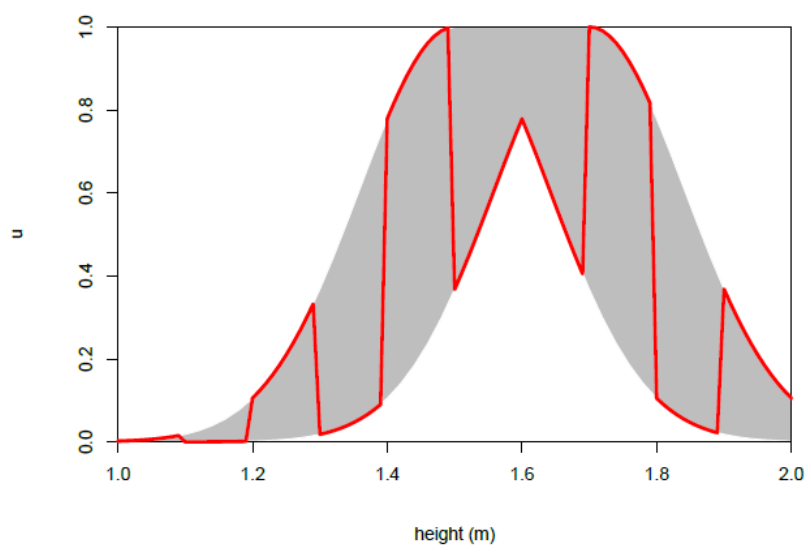

Fig. 4. An ES which is meaningless for the modelling of the concept of medium height (picture from [5])

is called generator set (GS). The only acceptable embedded sets (AES) of a CT2 set are represented by the ones obtained by shifting the GS MF of a fixed amount over the x-axis. In our example, the T1 set in Fig. 2 is the GS while the sets in Fig. 3 are some of its AESs. The FOU of our CT2 is obtained as the union of those AES and is therefore the one shown in Fig. 1.

A CT2 set is formally defined as follows:

Definition 7. A CT2 set denoted by $\widetilde{\mu}_{\delta}: X \times[0,1] \rightarrow[0,1]$ where, given a $\mu: X \rightarrow[0,1]$ (i.e. generator set) and $a$ continuous similarity relation $\delta: X \times X \rightarrow[0,1]$ and $a$ connected set $X$ such that $X \subseteq \mathbb{R}$ :

$$
\widetilde{\mu}_{\delta}(x, u)=\sup _{u=\mu(y)} \delta(x, y)
$$

We will use the notation $\widetilde{A}^{C T 2}$ to denote a CT2 set.

Definition 8. Given a CT2 set $\widetilde{A}^{C T 2}$ and its $T 1 G S \widetilde{G}$, the acceptable embedded sets $\underset{\widetilde{G}}{A} \mathrm{ES})$ of $\widetilde{A}^{C T 2}$ are all and only the T1 obtainable by shifting $\widetilde{G}$ within the FOU of $\widetilde{A}^{C T 2}$. More formally, given an embedded set $E$ :

$$
E \in A E S_{A} \Longleftrightarrow \exists c \in \mathbb{R}: \forall x \in X, \mu_{E}(x)=\mu_{G}(x-c)
$$

With universe of discourse $X$ being a connected subset of $R$

Note that CT2 sets can have secondary membership functions of any form - i.e. general constrained type- 2 sets (GCT2) - or secondary membership values that are always of height one - i.e. interval constrained type-2 sets (ICT2). Consequently, the latter case poses a limitation on the similarity relation, since it can only take 0 or 1 as values. The CT2 sets describes so far, significantly differ from the ones introduced in [7] in 2 key points:

- We generate the FOU as the union of the infinite T1 MFs obtained by horizontally shifting the $\mathrm{T} 1$ generator set in a fixed interval over the x-axis. Consequently, the FOU of a CT2 set can always be covered by the union of the acceptable embedded sets given in our representation even though all the acceptable embedded sets share the same shape.

- In some contexts, just considering non-convex and normal embedded sets may still be meaningless for the modelling of a concept. With our CT2 sets, instead, by fixing the shape of the acceptable embedded sets, we are keeping a very clear relation between the concept that was modelled by the $\mathrm{T} 1$ generator and its uncertain representation given by the IT2 CT2 set.

In the next two sections, we will focus on the analysis of ICT2 sets, exploring their structure to see how it can be used to develop a new inference process, setting the base of a future defuzzification algorithm that exploits the EASs of CT2 sets.

\section{BOUNDARY EVALUATION}

Since the FOU of a CT2 set is obtained as the union of all its AES (i.e. the T1 sets obtained by shifting the GS) it would be useful to establish a relation between the GS and the boundaries of the FOU in order to be able to evaluate the upper-bound and lower-bound MFs of the FOU given the GS function and the shifting steps.

Specifically, the upper-bound $\widetilde{A}_{u}^{C T 2}$ is obtained as the union of all the AES, i.e.:

$$
\mu_{\widetilde{A}_{u}^{C T 2}}(x)=\sup _{E \in A E S_{\widetilde{A}} C T 2} \mu_{E}(x)
$$

with $A E S_{\widetilde{A}^{C T 2}}$ being the set of all the AES for the $\widetilde{A}^{C T 2}$ set. All the $E \in A E S_{\widetilde{A}^{C T 2}}$ share the same shape since they are obtained by shifting the GS an infinite number of times within the given interval $\left[s t e p_{\min }, s t e p_{\max }\right]$, with $s t e p_{\min }$ and step $_{\max }$ being the minimum and maximum shifting steps used to generate the FOU. Therefore, (1) can be written as follows:

$$
\mu_{\widetilde{A}_{u}^{C T 2}}(x)=\sup _{\text {step }_{\min } \leq k \leq \text { step }_{\max }} \mu_{\widetilde{G S}}(x-k)
$$

For triangular and Gaussian GSs, the evaluation of the upper-bound is straightforward. In fact, we can separate the evaluation of $\mu_{\widetilde{A}_{u}^{C T 2}}(x)$ in 3 simple cases just by considering the GS, the x-axis coordinate $m_{x}$ of the peak point $m$ and the interval [step min $_{\text {, }}$ step $\left._{\max }\right]$ :

1) $x-$ step $_{\max } \leq m_{x} \leq x+$ step $_{\min }$

In this case, since by assumption $m_{x}$ is the $\mathrm{X}$-axis coordinate of the maximum point of the GS MF, from 2 we obtain:

$$
\mu_{\widetilde{A}_{u}^{C T 2}}(x)=\sup _{s t e p_{\min } \leq k \leq s t e p_{\max }} \mu_{\widetilde{G S}}(x-k)=\mu_{\widetilde{G S}}\left(m_{x}\right)
$$

2) $x-$ step $_{\max } \leq x+$ step $_{\min }<m_{x}$

That means that the MF, in the interval [step min $_{\text {, }}$ step $p_{\max }$ ], monotonically increases. Therefore $\mu_{\widetilde{A}_{u}^{C T 2}}\left(x_{2}\right) \geq \mu_{\widetilde{A}_{u}^{C T 2}}\left(x_{1}\right)$, with $x_{2} \geq x_{1}$. From (2), we deduce that the value of $k$ that maximises the argument of $\widetilde{A}_{u}^{C T 2}(x)$ is $k=s t e p_{\min }$, i.e. the minimum $\mu_{E}(x)$ is obtained when $E$ is the left-most AES of $\widetilde{A}^{C T 2}$. 
3) $m_{x}<x-$ step $_{\max } \leq x+$ step $_{\min }$

Here, the MF, in the interval [step $\min , s_{\text {max }}$ ], monotonically decreases, i.e. $\mu_{\widetilde{A}_{u}^{C T 2}}\left(x_{2}\right) \leq \mu_{\widetilde{A}_{u}^{C T 2}}\left(x_{1}\right)$, with $x_{2} \geq x_{1}$. From that, we deduce that the value of $k$ that maximises (2) is $k=s t e p_{\max }$, i.e. the membership value obtained from the right-most $\mathrm{AES}$ of $\widetilde{A}^{C T 2}$.

We can then conclude that to evaluate the upper-bound of a triangular or Gaussian CT2 set, we only need to consider the left-most and right-most AES, together with the peak point of the GS and the shifting steps.

For the evaluation of the lower-bound MF, (1) becomes:

$$
\mu_{\widetilde{A}_{l}^{C T 2}}(x)=\inf _{E \in A E S_{\widetilde{A} C T 2}} \mu_{E}(x)
$$

Therefore:

$$
\mu_{\widetilde{A}_{l}^{C T 2}}(x)=\inf _{s t e p_{\min } \leq k \leq s t e p_{\max }} \mu_{\widetilde{G S}}(x-k)=\mu_{\widetilde{G S}}\left(m_{x}\right)
$$

Now, we can apply the same reasoning we used for the upper-bound MF and obtain the same 3 different cases as for the upper-bound MF. Case 2) and 3) would be analogous to the case 2) and 3) of the upper-bound MF and this proves that in those scenarios we only need the left-most and right-most AES. The case 1), however, is slightly different.

Specifically, if we have $x-$ step $_{\max } \leq m_{x} \leq x+$ step $_{\min }$, we can split the interval $\left[x-\right.$ step $_{\max }, x+$ step $\left._{\min }\right]$ in 2 subintervals:

(i) $[x-$ step $\max , m]$

In this interval, the MF of our GS is increasing, therefore the value of $k$ that minimizes 5 is $k=x-s t e p_{\max }$, i.e. the minimum $\mu_{E}(x)$ is obtained when $E$ is equal to the left-most $\mathrm{AES} E_{L}$.

(ii) $\left.] m, x-s t e p_{\min }\right]$

In this interval, the MF of our GS is decreasing, therefore the value of $k$ that minimizes 5 is $k=x-s t e p_{\max }$, i.e. the minimum $\mu_{E}(x)$ is obtained when $E$ is equal to the right-most AES $E_{R}$.

We can then compute the MF values of these two minimizing points to evaluate the lower-bound, by taking the minimum of the two.

We can now individuate the minimum of the interval $[x-$ $\left.s t e p_{\max }, x+s t e p_{\min }\right]$ by taking the minimum of the two subintervals and then selecting the minimum of them as the global minimum:

$$
\mu_{\widetilde{A}_{l}^{C T 2}}(x)=\min \left(\mu_{E_{L}}(x), \mu_{E_{R}}(x)\right)
$$

where $E_{L}$ and $E_{R}$ are respectively the left-most and right-most AES.

This proves that we can fully determine the lower-bound of a CT2 set $\widetilde{A}^{C T 2}$ just by using the sets $E_{L}$ and $E_{R}$. These demonstrations can be easily extended to trapezoidal MFs, by considering the set of its peakpoint $M$ instead of $m$. Additionally, we speculate that just the knowledge of the leftmost and right-most AES together with the maximum and minimum points of the GS are sufficient to fully determine the FOU of a CT2 set with a GS of arbitrary shape.

\section{INFERENCE MODEL}

\section{A. Discretization}

Given the constrained structure of a CT2 and its unique definition of what an AES is, here we propose a new inference model that exploits their structure and sets some groundwork for future developments such as the design of a CT2 defuzzification algorithm. We define a CT2 fuzzy rule as a fuzzy rule in which all the MFs involved are CT2 MFs. A CT2 fuzzy system (CT2 FSM) instead, is a fuzzy system composed only by CT2 fuzzy rules.

Having defined what rules and systems are, we can now move to the inference process by considering a generic CT2 rule:

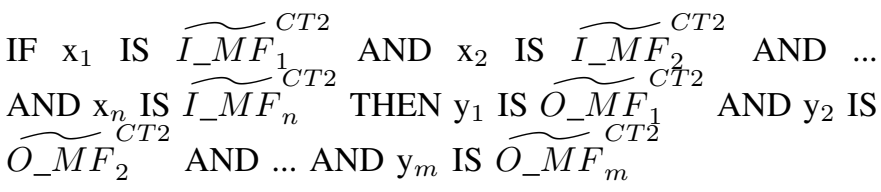

Each of these ${\widetilde{I_{-} M F_{i}}}_{i}{ }^{C T 2}$ and $\widetilde{O \_M F}_{j}{ }^{C T 2}$ have an infinite number of AES. However, to represent them in a computer, the continuous CT2 MF they belong to must be discretized. Hence, we propose a discretization that selects a fixed number of AES so that, for each discretization point $x$ :

$$
\sup _{E \in A E S_{\widetilde{A}}^{C T 2}} \mu_{E}(x)=\mu_{\widetilde{A}^{C T 2}}(x)
$$

with $\widetilde{A}^{C T 2}$ being the CT2 set to discretize and $A E S_{\widetilde{A}}^{C T 2}$ being the set of the selected AES with a finite cardinality.

To obtain such $A E S_{\widetilde{A}}^{C T 2}$, we include enough AES to be able to correctly solve all the 3 cases described in the Section IV for the boundaries evaluation.

We satisfy the cases 2) and 3) by including the left-most and right-most AES. For the case 3), instead, for each discretized value $x$ such that exists a peak-point $m$ of the GS that satisfies $x-$ step $_{\max } \leq m_{x} \leq x+$ step $_{\min }$, we add a new AES $E$ so that it's peak-point $m^{E}=x$. This process generates a finite number of AES within its FOU (Fig. 5), at most one per discretization point. Being a finite number, we can enumerate them from left to right by giving them an index.

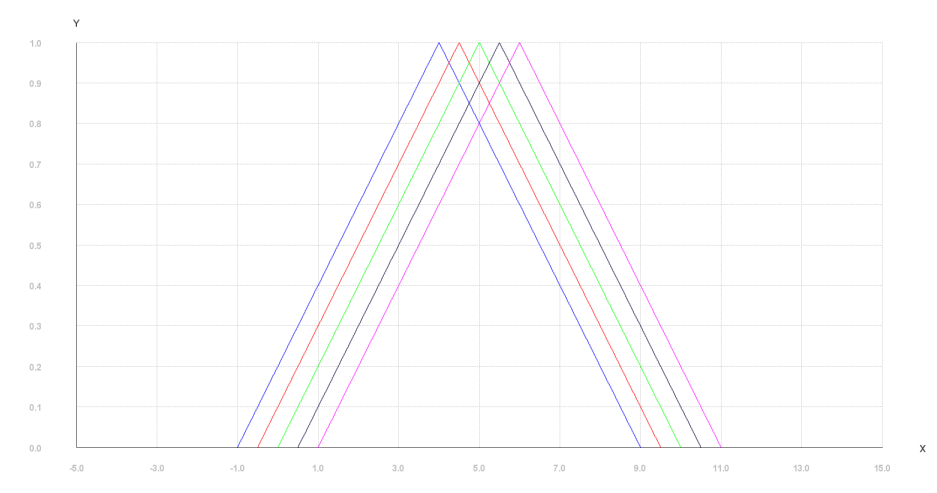

Fig. 5. Discretized triangular CT2 MF 


\section{B. Rule Conversion}

We now propose a novel inference model that exploits the CT2 structure and its AES. Informally, the goal is to convert each CT2 rule into a set of T1 rules by substituting each CT2 set with one of its AESs at a time, generating all the possible combinations.

Supposing we are converting the generic rule mentioned above, we obtain the following set of T1 rules (the apex notation specifies the index of the current AES of the CT2 set is belongs to):

IF $\mathrm{x}_{1}$ IS $\quad$ I_MF $\mathrm{MF}_{1}^{a}$ AND $\mathrm{x}_{2}$ IS $\quad \mathrm{I}_{-} \mathrm{MF}_{2}^{b}$ AND $\quad \ldots$ AND $\mathrm{x}_{n}$ IS I_MF ${ }_{n}^{c}$ THEN $\mathrm{y}_{1}$ IS O_MF $\mathrm{MF}_{1}^{d}$ AND $\mathrm{y}_{2}$ IS O_MF $\mathrm{MF}_{2}^{e}$ AND ... AND $\mathrm{y}_{m}$ IS O_MF ${ }_{m}^{f}$

with $a, b, c, d, e, f$ going from 1 to the number of AES the specific CT2 set they belong to contains. In other words, we are expanding one CT2 rule into a set of T1 rules by considering all the possible combinations of the AES of the CT2 sets. The inference result sets of each of these T1 rules can then be used to determine the upper-bound and the lower-bound functions of the new set resulting from the CT2 rule evaluation. An example of the output of this process is shown in Fig. 6.

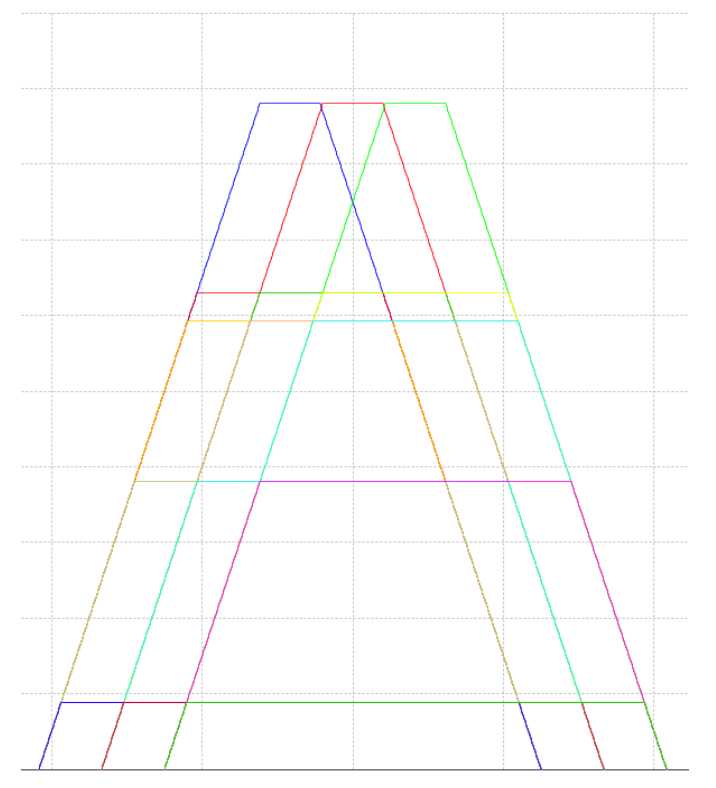

Fig. 6. Example of the inference output of the T1 rule sets generated from a CT2 fuzzy rule.

To obtain the FOU of the result of the CT2 inference, we need to evaluate the upper-bound and lower-bound MFs. We do so by preforming the union and the intersection, respectively, of all the T1 results of the inference. The result of this operation on the sets in Fig. 6 is shown in Fig. 7.

\section{ANALYSIS}

Now that we have defined how to carry out the CT2 inference, we focus on the analysis of the shapes this process

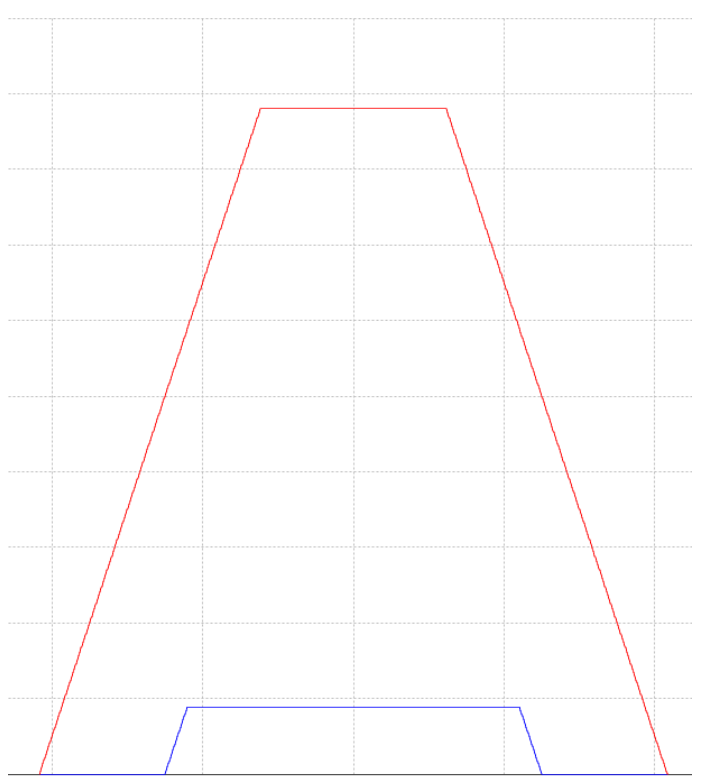

Fig. 7. Example of the upper-bound and lower-bound MFs obtained from the sets plotted in Fig. 6

produces on Gaussian, triangular and trapezoidal CT2. The first thing to notice is that none of the T1 sets obtained as described in the previous section is an AES of the inference result. In other words, no one of them could be used as a GS and be shifted by a given amount to obtain the full FOU. At the same time, also GS of the CT2 consequent MF before it was truncated by the inference process, doesn't keep the shape coherency concept that was expressed in the original formulation of the CT2 sets.

Even though they are not valid AES, by looking at Fig.6 it seems clear that there is some kind of relationship between them. By analyzing the conversion from a single CT2 rule to a set of T1 rules, we can see they all share the same shape, up to a certain point. In fact, by generating all the possible $\mathrm{T} 1$ rules from the combinations of the acceptable AES of the CT2 sets involved in a rule, each AES of the CT2 set used in the consequent part is paired with all the possible combinations of the AES of the antecedent CT2 sets. Therefore, each T1 inference output set, is simply obtained from an AES of the CT2 consequent set, cut at one of the possible heights computed by evaluating the antecedent part of the rule.

For example, suppose we have the following CT2 rule with 2 AES (indexes 1 and 2) per triangular CT2 set:

$$
\text { IF } x_{1} \text { is } \widetilde{A}^{C T 2} \text { AND } x_{2} \text { is } \widetilde{B}^{C T 2} \text { THEN y IS } \widetilde{C}^{C T 2}
$$

From this, we would obtain a total of six T1 rules. Three of them would have $C^{1}$ as a consequent T1 MF, while the other three rules would have $C^{2}$ instead. That means that each of these $C^{i}$ sets will be cut at (a maximum of) three different heights, obtained from the evaluation of the antecedent part of the three different rules. Additionally, the set of heights 
at which every AES of the CT2 consequent MF is cut, is the same for all the AES, since all the consequent AESs get paired with all the possible antecedent AEs combinations.

Having noticed this, we can now introduce the definition of layers (Fig.9) in the CT2 inference output:

Definition 9. A layer is the set of T1 sets obtained in the CT2 inference result that are generated by $T 1$ rules with the same antecedent component.

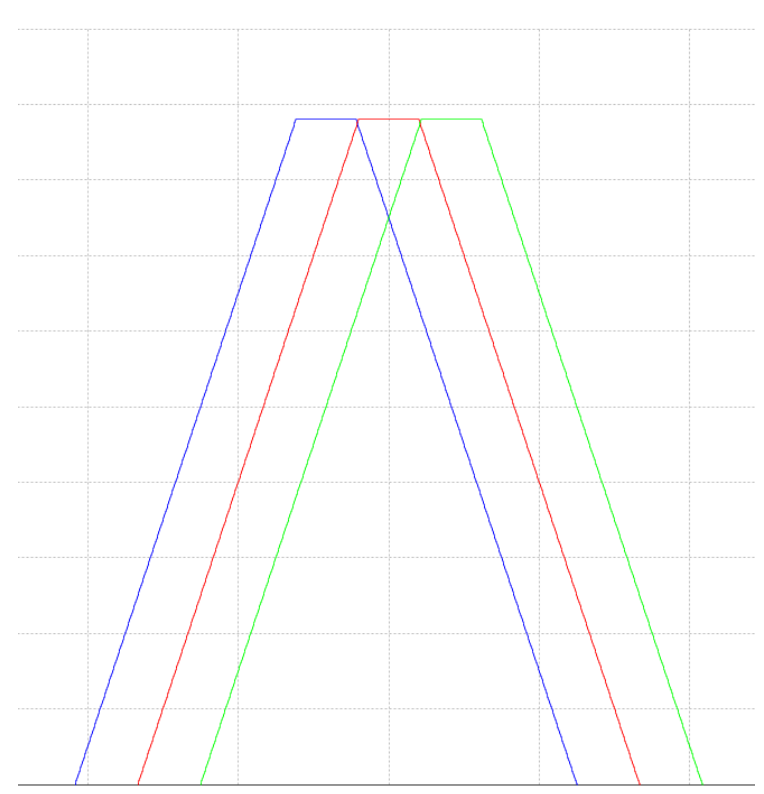

Fig. 8. Highest layer of the the inference result in Fig. 6

In Fig. 8 the highest layer of the inference output in Fig. 6 is shown. Since they are all obtained from the AESs of the same CT2 set and have been truncated at the same height, they all share the same shape. The only difference between them is the displacement on the X-axis. Therefore, they can be seen as AESs of a CT2 set, making each layer of the inference result a CT2 set.

We also notice that, for the shapes analyzed, the highest and the lowest layers share respectively the upper-bound and lower-bound MFs with the FOU of the whole inference result.

Furthermore, we anticipate that the concept of layers can be exploited for a new defuzzification process that is currently being developed.

\section{A. Comparison with Standard IT2 Inference}

After developing the CT2 inference, we compared it to the standard IT2 one, to see if they gave different results. To do so, we converted both CT2 MFs and rules into standard IT2 representation. Given a CT2 FS $\widetilde{A}^{C T 2}$ we can convert it to an IT2 set $A^{I}$ by evaluating the upper-bound and lower-bound MFs of $A^{C T 2}$ as described in Section IV, and then use those boundaries to define the FOU of $\widetilde{A}^{I}$.

We can now convert a CT2 fuzzy rule into an IT2 fuzzy just by replacing every CT2 set with the IT2 obtained from

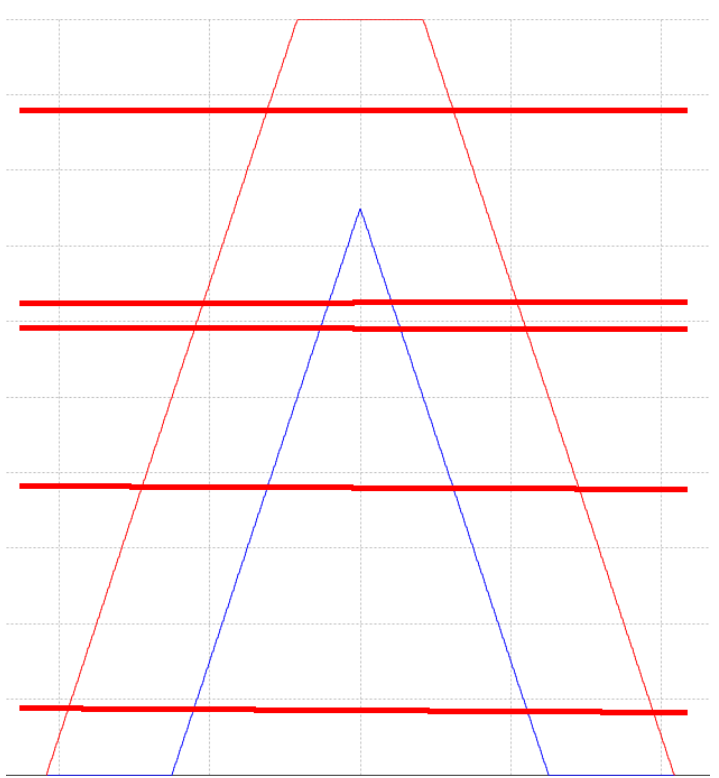

Fig. 9. The red lines indicate all the cuts that regenerate the layers shown in Fig.7

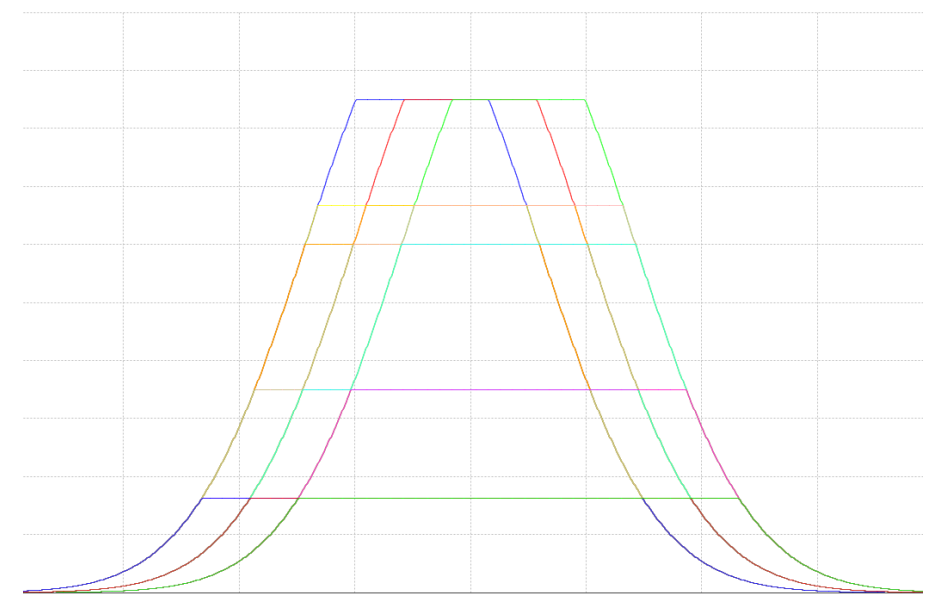

Fig. 10. CT2 inference result set with a Gaussian MF

the CT2 to IT2 conversion. In the experiments we ran with triangular, trapezoidal and Gaussian MFs, the CT2 inference proposed here and the standard IT2 inference process produced the same FOU, i.e. the same upper-bound and lower-bound MFs. Fig. 11 shows the IT2 inference result obtained from the conversion of the CT2 rules that generated the T1 MF in Fig. 10.

However, we believe that the additional information provided by the layers (especially by the highest and the lowest one), can be exploited for the defuzzification operation.

\section{CONCLUSION}

In this paper, we explored Interval CT2 sets and their constrained structure, focusing on MFs with Gaussian, trapezoidal 


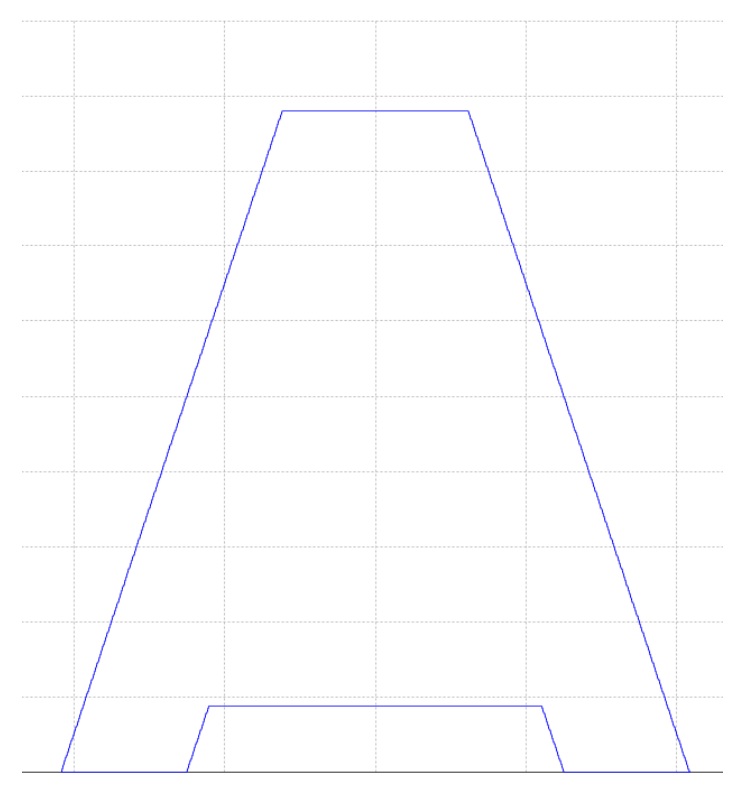

Fig. 11. IT2 inference result from the IT2 rule obtained from the $\mathrm{Ct} 2$ rules that generated Fig. 6

and triangular shape and establishing some groundwork for a future defuzzification method and practical use.

By analysing the definition of CT2s and their AESs, we showed how the upper-bound and lower-bound MFs (and therefore the FOU) can be obtained just by taking into account the left-most and right-most AES, together with the maximum points of the GS and the shifting steps used to generate the FOU. We also described a possible discretization method, based on the selection of a fixed number of the AES from the infinite amount that generate the FOU.

We then moved to the introduction of a new inference process (CT2 inference) that uses the AES obtained from the discretization. This novel model, is based on the conversion of CT2 rules into a set of T1 rules that generates the inference result boundaries. We pointed out how none of those T1 rules, however, generates valid GS for the inference result set. We also introduced the definition of layers, and showed how each one of them represents a valid CT2 set. Additionally, we anticipated that the concept of layers can be exploited for a new defuzzification method that is currently under development.

Finally, we showed how both the standard IT2 inference process and the CT2 one proposed here, generated the same FOU for CT2 sets with Gaussian, triangular and trapezoidal GS.

In future work, we plan on developing a novel defuzzification algorithm that can be used for CT2 system and we also plan on extending and generalizing the preliminary work showed here so that in can be applied to MFs of arbitrary shape.

\section{ACKNOWLEDGEMENTS}

Some of the MFs shown in the pictures of this paper, have been obtained using the Java-based fuzzy toolbox Juzzy [10].

\section{REFERENCES}

[1] L. Zadeh, "Fuzzy sets," Information and Control, vol. 8, no. 3, pp. 338 $-353,1965$.

[2] L. A. Zadeh, "Fuzzy logic = computing with words," IEEE Transactions on Fuzzy Systems, vol. 4, no. 2, pp. 103-111, May 1996.

[3] L. Zadeh, "The concept of a linguistic variable and its application to approximate reasoningi," Information Sciences, vol. 8, no. 3, pp. 199 249, 1975.

[4] J. M. Mendel, R. I. John, and F. Liu, "Interval type-2 fuzzy logic systems made simple," IEEE Transactions on Fuzzy Systems, vol. 14, no. 6, pp. 808-821, Dec 2006.

[5] J. M. Garibaldi and S. Guadarrama, "Constrained type-2 fuzzy sets," in 2011 IEEE Symposium on Advances in Type-2 Fuzzy Logic Systems (T2FUZZ), April 2011, pp. 66-73.

[6] N. N. Karnik and J. M. Mendel, "Centroid of a type-2 fuzzy set," Information Sciences, vol. 132, no. 1, pp. 195 - 220, 2001.

[7] D. Wu, "A constrained representation theorem for interval type-2 fuzzy sets using convex and normal embedded type-1 fuzzy sets, and its application to centroid computation," 042018.

[8] A. Pradera, E. Trillas, S. Guadarrama, and E. Renedo, On Fuzzy Set Theories. Berlin, Heidelberg: Springer Berlin Heidelberg, 2007, pp. $15-47$.

[9] J. M. Garibaldi, M. Jaroszewski, and S. Musikasuwan, "Nonstationary fuzzy sets," IEEE Transactions on Fuzzy Systems, vol. 16, no. 4, pp. 1072-1086, Aug 2008.

[10] C. Wagner, "Juzzy - a java based toolkit for type-2 fuzzy logic," in 2013 IEEE Symposium on Advances in Type-2 Fuzzy Logic Systems (T2FUZZ), April 2013, pp. 45-52. 